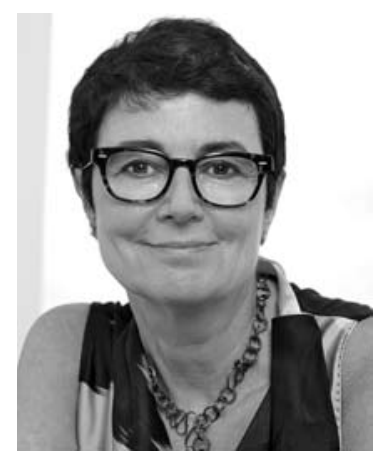

\title{
A Conversation with Titia de Lange
}

\author{
INTERVIEWER: GuY RIDDIHOUGH \\ Senior Editor, Science
}

Titia de Lange is Director of the Anderson Center for Cancer Research and the Leon Hess Professor at the Laboratory for Cell Biology and Genetics at Rockefeller University.

Guy Riddihough: Could you talk about problems that eukaryotic chromosomes have at their ends?

Dr. de Lange: Eukaryotes all have linear chromosomes and it's not clear why or how that happened, because prokaryotes have circular chromosomes and they do fine with them: It's better for DNA replication, but it's also better because both prokaryotes and eukaryotes have a system to look for DNA damage. This system constantly monitors the DNA for breaks that need to be repaired. When our cells experience DNA breaks, the cells stop the cell cycle so that the repair can take place. The system is really quite vigilant; it's thought that it can find a single DNA break in the genome.

The problem with linear eukaryotic chromosomes is that they have these free ends. All hell would break loose if the repair system recognized these natural chromosome ends as signs of DNA damage, because cells would arrest in their cell cycle and might try to repair these ends by ligating one chromosome end to another. Because the DNA damage response system was already in prokaryotes when linear chromosomes evolved, the cells had to evolve a solution to this problem.

Guy Riddihough: What is that solution?

Dr. de Lange: That was identified by Barbara McClintock and Hermann Müller, who realized that the end of a chromosome is different from other parts of the chromosome. They named this section the "telomere" from "telos meros": Greek for "end part." The telomere identified for the cells that this was a natural chromosome end: Don't mess with it.

In the late 1970s and early 1980s Liz Blackburn working with Joe Gall and others found that telomeres have a very specific, very boring DNA sequence. Human telomeres are made up of about 10,000 base pairs that go TTAGGG, TTAGGG, over and over again. It's like a Gregorian chant at the end of the symphony of the genome. Yet this is the sequence that allows a cell to say "This chromosome end is natural; there's no need to repair it."
Guy Riddihough: So, the end is disguised from the repair machinery that's present in all prokaryotes and eukaryotes. In that transition from circular to linear chromosomes, there has to be a mechanism to basically secrete it away somewhere. What is the nature of that machinery or system?

Dr. de Lange: I think during evolution the first chromosome ends were sloppy, and at some point telomerase evolved. That's the enzyme that makes the repeat sequence. Telomerase also solves another problem associated with chromosome ends, which is that you can't fully replicate the end of a linear DNA. The replication machinery evolved in the context of circular genomes, so it never experienced evolutionary pressure to deal with a chromosomal end. It can't finish the job at the end and telomerase is one of the ways to solve this problem.

Guy Riddihough: Without telomerase, you couldn't replicate the full end, and the chromosomes would get shorter and shorter after each round of replication.

Dr. de Lange: That's what you see when you knock out telomerase in yeast, which first was done by Vicki Lundblad and Jack Szostak, or in mouse cells, which was done by Carol Greider and Ron DePinho. The chromosome ends gradually get shorter. You need telomerase to counteract that. We do that in our germline; we keep our telomeres intact. In principle, there are many solutions to this end replication problem. Every virus with a linear genome has this problem and they have different solutions: For example, adenovirus has a protein hanging onto the end that can prime DNA synthesis. The great thing about telomerase is that it standardizes the sequence at every chromosome end. Once you evolve telomerase as a solution for the end replication problem, all telomeres have the same equally boring TTAGGG repeat sequence.

At that point in evolution, it became possible to evolve proteins that recognize the sequence and bind to all telomeres. These proteins became essential for the protection. In our telomeres there is a complex called shelterin, composed of just six proteins, seven in the mouse. 
It's very specific for the telomere sequence and they do an amazing job of hiding those TTAGGG repeat ends from the DNA damage response.

Guy Riddihough: If you remove the shelterin complex, what are all of the different things that the end of the chromosome could be exposed to in terms of inappropriate processing?

Dr. de Lange: It's a nightmare. Recently, we took the whole complex off telomeres and found there are six or seven pathways that get activated. The ATM and ATR kinases - the two main transducers of DNA damagebecome active and enforce cell cycle arrest. Then the repair machinery starts acting on these ends. Both the classic and alternative forms of the nonhomologous end-joining pathway are activated. These are ligation pathways that glue the ends of all chromosomes together. There's also homologous recombination, where telomeres start invading each other, and resection, which is when the nucleases that trim the broken ends of damaged DNA to prepare them for homologous recombination start to eat the telomere away. So shelterin has just six proteins in it, but it protects the telomeres from all these different pathways.

We used to think of telomeres as a sort of molecular condom, with shelterin forming a cap over the telomere and protecting it. Instead, shelterin compartmentalizes its functions. Different proteins take care of different pathways. The question now is how they function. For example, the ATM kinase is activated by a protein complex that recognizes DNA ends regardless of the sequence, so how does shelterin protect this DNA end from being recognized?

Guy Riddihough: In addition to the shelterin complex, does the telomere end also have a specific structure?

Dr. de Lange: We were working with Jack Griffith, an electron microscopist at the University of North Carolina, when we realized that we had no idea what the actual structure of a telomere was. Jack managed to look at these structures and instead of a linear sequence he found these gigantic loops. The chromosome end wasn't out, it was tucked in. We thought maybe the ends were protected because they were hidden inside the telomere where the ATM kinase and nonhomologous end-joining pathways - the two pathways that will activate if they detect a DNA end-couldn't detect them.

We also found that TRF2 (telomeric repeat-binding factor 2), which is a component of the shelterin complex, could form loops by itself in vitro. Not very well, but it could do it. We thought that TRF2 might be responsible for tucking in the end of the telomere. The key experiment to test that model was to delete TRF2 from the cells and see whether the loops disappeared. We tried that for about 14 years. It was an impossible experiment to do by electron microscopy. You can't tell what the sequence of a chromosome end is by looking at it under the electron microscope, so we needed to purify telomeric DNA away from the rest of the genome first. To do that, we had to run a Bio-Gel column that was a meter long. This is not a quantitative technique. If you do a deletion experiment, you want to be able to count molecules, so we were stuck. We couldn't test our model. In the meantime, the model became dogma in the field. Everybody wrote in their introductions that TRF2 makes t-loops and protects telomeres. I became desperate because if we ever proved this, we wouldn't be able to publish it because everybody would say "We already knew this."

We finally solved the problem using STORM (stochastic optical reconstruction microscopy) imaging, a type of superresolution imaging developed by Xiaowei Zhuang at Harvard that allowed us to look at telomeres directly in cells without purifying them. Unfortunately, when we did STORM imaging, we didn't see loops; the telomeres were these tiny little nubbins. You couldn't see any detailed structure. A postdoc in my lab-Ylli Doksani-spent 2 years developing a technique by which he could relax the telomeres so that we could see the loops in cells. When we imaged cells treated with his technique we found that the loops were opened in the cells lacking TRF2. It took 15 years, but we were finally able to confirm our model.

Guy Riddihough: Another thing that's interesting and perhaps a little more controversial about telomeres is their role in aging.

Dr. de Lange: It's clear that telomeres shorten with cell divisions and we go through a lot of cell divisions, different numbers of divisions in different organs, as we age. Carol Greider was the first to provide data on that at Cold Spring Harbor in the early 90s. The question is, does it matter? Do we age because of our telomeres? You really need a genetic experiment to prove that. Carol and Ron DePinho knocked out telomerase in mice and found that mouse telomeres shorten, just as much as human telomeres do. Mice start out with fairly long telomeres, so you have to wait for a long time, breeding them over generations. Eventually, they show aging phenotypes and lots of things go wrong in these mice. They become sterile, they have liver problems, and so on.

About 15 years ago, there was a breakthrough in this area with regard to human biology. Kathy Collins was the first to show that there is a human disease called "dyskeratosis congenita" that is caused by insufficient telomerase. These people have defects either in the telomerase complex itself, an RNA component, a reverse transcriptase, or in the biogenesis factors, and they end up with lowered levels of telomerase. Not absent, but lowered. Over generations, you see telomeres getting shorter in these families and the onset of the disease occurs earlier and earlier. This is clearly caused by telomere shortening. The main symptom is bone marrow failure. These people need to be treated with bone marrow transplants. Those that survive longer develop other problems: liver cirrhosis, lung fibrosis, serious disease phenotypes. On the other hand, it's not progeria; these are not old people at the age of 4. It's not a general aging phenotype, but it tells you what you can expect as a result of telomere shortening. It's reasonable to assume that telomere shortening affects bone marrow, lungs, and liver as you age, but 


\section{A CONVERSATION WITH TITIA DE LANGE}

probably not the brain. These people don't develop Parkinson's or Alzheimer's, they don't get cardiovascular disease, they don't get wrinkles. Telomeres probably do determine aspects of aging, but it's organ-dependent.

Guy Riddihough: There's a link with cancer and telomeres as well, isn't there?

Dr. de Lange: There's an important link we've known about since 1994. Telomere shortening occurs in normal human cells. At some point, the telomeres get so short that the protection doesn't work anymore and the cells think they have broken ends and stop dividing. This functions as a great tumor-suppressive system. Recent genetic data argues that we are protected from cancer to a great degree by telomere shortening. But like any tumor-suppressive pathway, it can fail and cancer cells find a way around this. One way they do that is by reactivating telomerase, the enzyme that maintains telomeric DNA. Most human cancers have telomerase activity. For the longest time, we didn't know whether this was actually a selected event during tumorigenesis or just a side effect. Cancer cells have certain stem cell features, and stem cells have telomerase, so it was possible the telomerase was just a consequence of that "stem cell-like" aspect of the cancer.

Recently, it was found that telomerase in cancer is activated by mutations in the promoter for the telomerase reverse transcriptase. Levi Garraway and Rajiv Kumar's groups found point mutations in the promoter that creates transcription factor binding sites. Telomerase becomes active, whereas normally it's silenced. This is how the enzyme becomes up-regulated. This is a highly selectedfor event and it confirms that telomere shortening is something that most cancers worry about because they select for something that acts against this pathway. It's become clear that in many tumors, these promoter mutations are a frequent event. There are probably other mechanisms by which telomerase is activated. 


\section{$\$_{\text {CSH }}^{\infty}$ Cold Spring Harbor Symposia SYMPOSIA On Quantitative Biology}

\section{A Conversation with Titia de Lange}

Cold Spring Harb Symp Quant Biol 2015 80: 311-313

Access the most recent version at doi:10.1101/sqb.2015.80.029918

\section{License}

Email Alerting Receive free email alerts when new articles cite this article - sign up in Service the box at the top right corner of the article or click here. 